\title{
The Effectiveness of a Course on Patient Safety Management on the Patient Safety Competencies by Final Year Bachelor of Nursing Students in the Netherlands
}

\author{
Timmermans Olaf ${ }^{1,2 *}$, De Vos Mieke ${ }^{3}$ and Franck Eric ${ }^{1,4}$ \\ ${ }^{1}$ Centre for Research and Innovation in Care, University Antwerp, Antwerp, Belgium \\ ${ }^{2}$ Professorship Healthy Region, HZ University of Applied Sciences, Vlissingen, The Netherlands \\ ${ }^{3}$ Vesalius University College, Ghent, Belgium \\ ${ }^{4}$ Karel de Grote University College, Department of Health Care, Antwerp, Belgium
}

\begin{abstract}
Background: Enhancing competencies of patient safety and quality of care is an actual and important issue in Bachelor of Nursing education. In 2010, the Dutch ministry of health adopted ten themes of patient safety and developed six competences for patient safe nursing care. Nursing schools integrated these competences in their Bachelor of nursing programs. This study evaluates the progress of undergraduate nursing students on the six competences, before and after a course on the management of innovation and policy-making of safety management.
\end{abstract}

Methods: This study used a quantitative, quasi-experimental intervention survey design in last year Bachelor of nursing students ( $\mathrm{n}=55)$ of one Nursing school in the Netherlands. Students scored self-reported levels of mastery on all six patient safety competencies on a 10-point visual analogue scale. Differences in the pre- and posttest were considered using the Paired Sample T-test.

Results: Significant differences were detected in the total scores of the perceived level of mastery of all six patient safety competencies before and after the course. Three individual competencies showed significant differences: 'contribute to a culture of patient safety', 'working in teams' and 'optimize human and environmental factors'.

Conclusion: The course positively affected the perceived level of mastery of the effectiveness of a patient safety culture, more effective communication and optimizing human and environmental factors. Overall, the course had a significant effect on the total score in management of the patient safety competencies.

\section{Introduction}

Healthcare systems are complex organizations with often unpredictable risks, that have an impact on safe delivery of patient care $[1,2]$. It has been suggested that nurses are more likely than any other healthcare professionals to recognize, intercept and correct errors that are often life threatening $[1,3,4]$. Nurses are at the centre of patient care and therefore essential key figures of quality improvement in healthcare organisations [5]. Patient safety is becoming majorly important in delivering healthcare and education, both on a national and international level [2,6]. Due to the publications 'To Err Is Human' and 'Crossing the Quality Chasm', produced by the Institute of Medicine (IOM), the healthcare system shows an increased focus on patient safety $[4,7,8]$. The IOM defined patient safety as freedom from accidental injury. Furthermore, patient safety is defined as the prevention of unnecessary harm to patients, by use of best practices [7,9]. Tella et al. [10] defined patient safety as minimizing a patient's exposure to hazards and near-misses. Hereby a hazard is defined as an intervention or an event that has the potential to cause harm to a patient. Most known hazards are a lack of communication and verification of allergies and inadequate hand-hygiene. A near miss or 'good catch' is an event, situation, or incident that could have caused adverse consequences and harmed a patient, but did not $[5,10]$. Most common 'near misses' are found in medication administration [10,11].
Medication errors mostly are preventable events that may cause or lead to inappropriate medication use or patient harm while the medication is in the control of the health- care professional, patient or consumer [12]. Medication errors have been found to be due to the result of human factors, whereby performance and knowledge deficiencies were found to be two major factors for errors among nurses [5]. Adverse events are defined as "unintended consequences that are caused by the action or inaction of a caregiver and/or healthcare system and involve harm to the patient that is so serious that there is temporary or permanent disability or even the patient's death" [13]. For example: a rash caused by an antibiotic or a ventricular tachycardia due to placement of a central venous catheter tip in the right ventricle. Studies still underwrite human factors as most important causes for hazards, near misses and adverse events.

Correspondence to: Timmermans Olaf, Centre for Research and Innovation in Care, University Antwerp, Antwerp, Belgium \& Professorship Healthy Region, HZ University of Applied Sciences, Vlissingen, The Netherlands, E-mail: olaf. timmermans@uantwerpen.be

Key words: education patient safety, patient safety competencies, nursing, baccalaureate, healthcare education

Received: September 30, 2017; Accepted: October 28, 2017; Published: October 31,2017 
This is why health care professionals should have basic knowledge, attitudes and skills to assure patient safety $[2,14$.

To reduce unsafe nursing care, increased emphasis, knowledge and skills on patient safety in nursing education are imperative $[4,11,15]$.

\section{Background}

The World Health Organization (WHO) highlighted the increase incidence of adverse events, including adverse drug events and nosocomial infections. 'Adverse events' research came to prominence when the Institute of Medicine reported that in-hospital medical errors alone killed more people than HIV/AIDS, breast cancer or motor vehicle accidents. Adverse drug events (ADEs) account for nearly 100 000 emergency department visits and 700000 hospitalisations each year in the USA, 5-8\% of unplanned hospital admissions in the UK, and 2-3\% of all admissions in Australia. Medication errors and health care related adverse events occurs in $8-12 \%$ of all hospital admissions in the European Union Member States [12]. In 2011, the WHO launched the Evidence-based recommendations for embedding patient safety in nursing education based on the WHO Multiple- professional Patient Safety Curriculum Guide $[2,16]$. In addition, a greater emphasis is being placed on the integration of patient safety competencies into health professional education $[9,10]$ (Table 1).

In order to develop optimal nursing competency in patient safety and quality, an analysis of the current nursing program in terms of how patient safety education is delivered and of the present state of nursing students' competencies are needed prior to the design and reconstruction of a nursing program based on patient safety. In contrast to Ranjbar and Emami Z [17], nursing universities are integrating the WHO competencies in nursing curricula to provide education on patient safety.

Lukewich et al. [9] studied the course of nursing students' confidence in sociocultural aspects of patient safety within the classroom and clinical setting across the years of nursing education and found an increase in the following patient safety competencies: clinical safety $(n=4)$; managing safety risks $(n=3)$; human and environmental factors $(n=3)$ and adverse events $(n=3)(p<0.01)$. Also, they observed a significantly decrease of working in teams $(n=6)$ and communicating $(\mathrm{n}=3)$ during the years of nursing education $(\mathrm{p}<0.01)$ [9].

To describe the required educational interventions, several models based on the required competencies of nursing-students have been developed [12]. For example, patient safety competencies could be assessed by final year baccalaureate nursing students by the self-reported Health Professional Education in Patient Safety Survey (H-PEPSS). This framework underlines the sociocultural aspects of patient safety including culture $(n=4)$, teamwork $(n=6)$, communication $(n=3)$, managing risk $(n=3)$ and understanding human factors $(n=3)$ [18]. In comparison to the H-PEPPS model, that is merely based on selfassessment, the Objective Structured Clinical Examination (OSCE) contains an external assessment of knowledge, skills and attitudes on during reality based simulation context, based on the Canadian Patient Safety Institute (CPSI) Safety Competencies (2009). These competencies

Table 1. The Patient Safety Curriculum Guide contains six patient safety competencies developed by the World Health Organization.

Domain 1: Contribute to a culture of patient safety $(n=9)$

Domain 2: Work in teams for Patient Safety $(n=8)$

Domain 3: Communicate effectively for patient safety $(n=6)$

Domain 4: Manage safety risks $(n=6)$

Domain 5: Optimize human and environmental factors $(n=6)$

Domain 6: Recognize, respond to and disclose adverse events $(n=8)$ were classified by the patient safety competencies of the World Health Organization. The OSCE used Millers framework for clinical assessment of the six patient safety competencies $[19,20]$. In addition, the OSCE includes awareness of the culture and the complexity of the healthcare system, as well as teamwork and the relevance of learning from skills $[19,21]$. Similar to the patient safety framework of the WHO, the Quality and Safety Education for Nurses (QSEN) includes six quality and patient safety domains, more specifically patient-centred care, evidence based practice, quality improvement, informatics, patient safety, teamwork and collaboration [1,2,10,22-26]. These domains were explicitly established to create inter-professional education [23]. Finally, knowledge regarding patient safety could be measured by the Health Care Professionals Patient Safety Assessment Curriculum Survey (HPPSACS). These instrument assesses four factors; willingness to disclose errors; recognitions and management of medical errors; the perceived interprofessional context, support and understanding for improving patient safety. Moreover, this instrument provides the opportunity to monitor the development of awareness, skills, and attitudes towards patient safety education $[1,25,27]$.

Embedding patient safety competencies in the current curriculum can be done by development of a particular course on patient safety wherein the emphasis is on the organization system and human factors, effective teamwork and optimal communication [6,10,23,24,26-28]. Moreover, to appeal for a 'blame and shame free' culture it is essential to learn nursing students how to handle and prevent adverse events and to interference in reporting errors and coping with the fear of legal prosecution $[3,6,9]$. Although reporting errors and learning from the adverse events are vital, disclosing mistakes can be frightening for students. In absence of any positive feedback and the attention being mainly on an individuals' performance and mistakes, students are anxious of being punished by the health care staff or being judged as incompetent [2]. Culture also affects perceptions of patient safety, especially the role of culture in the readiness of individuals to speak up when there is a threat to patient safety and the shame and blame culture type. Quality improvements in patient safety often focus on changing culture [29]. These environments should enhance learning form mistakes by analysing what happened to gain a full understanding of the encounter without triggering a judgmental behaviour [22]. In order to acquire a level of mastery on patient safety competencies, nursing education uses appropriate teaching methods to supply the fundamental components of patient safety [1,3,4,8-10,27]. Patient safety is a part of daily practice, however, is not yet included in all nursing education curricula [3]. This results in a lack of knowledge and skills concerning patient safety [28]. Several authors state that nursing curricula therefore should be adjusted [10,26,27]. In essence, nursing students must learn the fundamentals of patient safety, learn how to prevent and handle adverse events from the very beginning of their nursing studies $[6,10,25]$. In 2010, the Dutch ministry of health adopted ten themes of patient safety and developed six competences for patient safe nursing care.

Nursing schools integrated these competences in their Bachelor of Nursing programs. In the current nursing curricula of the regarding educational institution, nursing students take up a 7.5 ECTS credits (European Credit Transfer System) course on management, innovation and policy of patient safety. In the second semester, the students use the obtained competences in an assignment in clinical practice.

In the course innovation, policy and management of patient safety, students first learn a systematic way of risk assessment and to 
detect an issue on patient-safety and its' barriers in nursing care. Then these barriers - inclusive prioritization - are framed in the perspective of quality of care. Finally, from innovation and team management, students learn to find solutions for the barriers to implement solutions in the clinical setting and to measure their effects. This study aims to determine the effects of this course on the perceived level of mastery on patient safety competences by final year baccalaureate nursing students in one Nursing School in the Netherlands.

\section{Methods}

A quantitative, quasi-experimental intervention survey was conducted to gather self-reported data from last year baccalaureate nursing students in the Netherlands. The allocation of respondents relies on no randomization. This study was based on a pre and post measurement.

\section{Setting and respondents}

In the cohort 2014-2015, all final year baccalaureate nursing students $(n=59)$ enrolled in a nursing program in one Nursing School at an University of Applied Sciences in the Netherlands were eligible to participate in this study.

All final year students, who were enrolled on a degree pathway from each of the following three nursing specializations: general hospital nursing, psychiatric nursing and community nursing were invited to participate in the study. We observed a drop-out in the post-test, resulting in a total sample size of 55 final year nursing students $(n=55)$.

\section{Ethical considerations}

Approval for this study was granted by the administration of the educational institution. Furthermore, an informed consent form with information about the aim and design of the study was given to the respondents.

Furthermore, participation was done on a voluntary basis and could be refused. Students had to sign the prior to their participation. Finally, privacy protection was guaranteed by anonymizing the database.

\section{Data gathering}

Data were collected in September 2014 and January 2015. The used questionnaire was based on the WHO's patient safety competency framework. Within those framework, the questionnaire contained 43 items divided into the six competences: $1 /$ Contribute to a Culture of Patient Safety $(n=9) ; 2 /$ Working in teams for patient safety $(n=8) ; 3$ / Communicate effectively for patient safety $(n=6)$; 4 /Manage safety risks $(n=6) ; 5 / O p t i m i s e$ human and environmental factors $(n=6)$ and 6/Recognise, respond to and disclose adverse events $(n=8)$ [30]. Respondents were asked to rate their perceived mastery level on a scale ranging from 0 (no mastery at all) to 10 (high level of mastery) before and after the course on patient-safety.

In addition to the prior data, the questionnaire also included demographic data and specific educational data: the highest degree; the nursing specialization; the combination nursing and midwifery; internship in the clinical setting, in a public clinical setting or abroad.

\section{Statistical analysis}

Data were analysed using Statistical Package for the Social Sciences (IBM SPSS Statistics 23.0. Mac OS X version). New variables $(n=15)$ were made (i.e. subscales, differences and total scores) which were related to the level of mastery of the patient safety competencies in the pre-test and post-test.
All variables were coded and checked for the normality of the distributions. Based on detected normal distribution, parametric statistics were handled. Cronbach's alpha was used to assess the internal reliability of the constructed subscales of all six competencies and the total, overall scale and ranged from 0.64-0.93 (pre-test) and $0.68-0.92$ (post -test).

Descriptive statistics were performed for an overview of the demographic and educational data. In addition, continuous data are presented as mean, range and standard deviations and categorical data are presented as percentages and numbers. Paired Sample T- test were conducted to analyse differences in scores of the pre and postmeasurement.

All p values were based on two- tailed tests of significance. Statistical significance was considered with $\mathrm{p}<0.05$.

\section{Results}

Table 2 presents an overview of the respondents and descriptive results.

The characteristics were classified into demographic and specific educational data.

The majority of the respondents (91\%) were enrolled in general nursing as specialization. Merely $16 \%$ did the combination of nursing and midwifery. The majority of the respondents (91\%) had already got internship in a clinical setting. Less than half (49\%) had done an internship abroad. $18 \%$ had done an internship in a public clinical setting. First, the total scores of the competences in patient safety were examined. The pre-test showed that the mean of the total score in the perceived level of mastery of all patient safety competencies was 7.07 at the start of the course. The mean total score increased after the course to 7.38 ( $\mathrm{p}=0.003$; Table 3) (Table 2).

Table 3 shows the competencies 'working in teams' (6.76), 'optimize human and environmental factors' (6.92), 'manage safety risks' (7.03) and 'contribute to a culture of patient safety' (7.03) presented lowest mean scores on perceived level of mastery in the pre-test. High mean scores were presented by the competencies 'communicate effectively' (7.46) and 'recognise, respond to and disclose adverse events' (7.21). In contrast, highest mean scores of the perceived level of mastery in the post-test were presented by the competencies 'effective communication on patient safety' (7.56), 'manage safety risks' (7.20), 'contribute to a culture of patient safety' (7.38) and 'management in optimize human and environmental factors' (7.29). The comparison of the pre and posttest mean scores is demonstrated in table 3. Mean scores of perceived mastery on the total scale of all six competencies ( $\mathrm{p}=.003)$, and the competencies 'contribution to a safety culture' ( $\mathrm{p}=0.038$ ), 'working in teams' $(\mathrm{p}<0.001)$ and 'optimise human and environmental factors' $(\mathrm{p}=0.038)$ were significant higher in the post-test (Table 3$)$.

\section{Discussion}

Patient safety is discussed as being elemental to quality. Moreover, patient safety and quality of care should be at the core of health care systems and processes and are inherently dependent upon nurses [5]. Nurses are in the position to ensure patient safety through monitoring, whereas nurses spend the most time with patients in the front line of care. In addition, nurses are the largest group of healthcare professionals [16]. Therefore it has been suggested that nurses are more likely than other health care professionals to recognize, intercept, and correct errors that are often life threatening [31]. 
Table 2. Characteristics of the respondents and descriptive results

\begin{tabular}{|l|l|l|}
\hline \multicolumn{2}{|l|}{ Demographic data N=55 } & $21(19-25)$ \\
\hline Age, y,mean (range) & Female & 91 \\
\hline Sex (\%) & Intermediate Vocational Education & 11 \\
\hline \multirow{2}{*}{ Degree (\%) } & $\begin{array}{l}\text { Higher General Secondary } \\
\text { Education }\end{array}$ & 71 \\
\cline { 2 - 3 } & Pre University Education & 18 \\
\hline Specific educational data & & \\
\hline Specialization (\%) & General nursing & 91 \\
\hline Combination nursing and midwifery (\%) & Yes & 9 \\
\hline Intership in a clinical setting (\%) & Yes & 16 \\
\hline Internship abroad (\%) & Yes & 91 \\
\hline Internship in a public clincal setting (\%) & Yes & 49 \\
\hline & & 18 \\
\hline
\end{tabular}

Table 3. Differences in subscales and total scores of the pre and post-test perceived level of mastery of patient safety competencies.

\begin{tabular}{|l|l|l|l|}
\hline \multirow{2}{*}{$\begin{array}{l}\text { Patient safety competencies } \\
\text { (n=6) }\end{array}$} & $\begin{array}{l}\text { T0 level of } \\
\text { mastery of } \\
\text { patient safety }\end{array}$ & $\begin{array}{l}\text { T1 level of } \\
\text { mastery of } \\
\text { patient safety }\end{array}$ & \multirow{2}{*}{$\mathbf{P}^{\star}$} \\
\cline { 1 - 3 } & Mean (SD) & Mean (SD) & \\
\hline $\begin{array}{l}\text { Contribute to a culture of } \\
\text { patient safety (n=9) }\end{array}$ & $7.03(1.23)$ & $7.38(0.69)$ & $\mathbf{0 . 0 3 8}$ \\
\hline Working in teams (n=8) & $6.76(0.74)$ & $7.16(0.68)$ & $<\mathbf{0 . 0 0 1}$ \\
\hline Communicate effectively (n=6) & $7.46(0.61)$ & $7.56(0.63)$ & 0.257 \\
\hline Manage safety risks (n=6) & $7.03(0.65)$ & $7.20(0.58)$ & 0.111 \\
\hline $\begin{array}{l}\text { Optimise human and } \\
\text { environmental factors (n=6) }\end{array}$ & $6.92(1.23)$ & $7.29(0.62)$ & $\mathbf{0 . 0 3 8}$ \\
\hline $\begin{array}{l}\text { Recognise, respond to and } \\
\text { disclosure adverse events (n=8) }\end{array}$ & $7.21(0.82)$ & $7.37(0.61)$ & 0.133 \\
\hline Total score (n=55) & $7.07(0.66)$ & $7.33(0.51)$ & $\mathbf{0 . 0 0 3}$ \\
\hline
\end{tabular}

"Paired Sample T-test

Given the diverse scope of work within the nursing profession, to produce nurses competent in patient safety, education in patient safety has to be lectured from the first year and should be continued throughout their nursing education. Nursing students need basic knowledge and skills to keep patients safe in continuously changing health care environments [23]. Patient safety education in nursing programs is the first step toward ensuring patient safety and quality in clinical practice $[16,31]$. This paradigm shift will enable student nurses to think more critically and clinically, and to achieve greater insights [8]. Education has a pivotal role in patient safety through the development of knowledge, attitudes, values, skills and practice. It also raises awareness of patient safety incidents, their causes and prevention. Education establishes knowledge and skills required to promote and improve safe patient care [31].

Learning systematically from errors in a supportive environment and having systems-based approaches to ensure patient safety are essential elements for nursing students learning about safe practice [2].

This study highlighted the patient safety competencies in the final year of the Bachelor Nursing program within one University of Applied Sciences in the Netherlands. Results show that the course Innovation, Policy and Safety management resulted in a significantly increase in the perceived level of mastery of the competencies 'contribution to a culture of patient safety', 'working in teams' and 'optimising human and environmental factors'. Overall, there is a significant effect on the total score of the perceived level of mastery of patient safety competencies in the post test. This finding affirms the result of another study.
Cruz et al. [31] argues that respondents reported significantly higher confidence in patient safety learning in the classroom setting in line with the patient safety competencies, 'working in teams', 'understanding human and environmental factors', 'culture of safety' and 'managing risk'. It implies that nursing students are more confident in what they learn about patient safety in the classroom than in the clinical setting. As a result, the role and the quality of the relationship between students and nurse instructors in the clinical settings are important. Problems may arise from specific contextual factors, for instance lack of time for teaching, conflicting relationships within the team, and the consolidated practice of inadequate procedures $[2,22]$.

There is, however, a lack of a general perception within all years of the Bachelor Nursing education [9]. Numerous studies have been conducted examining patient safety competence in the classroom and clinical settings. These studies suggest a strong impact of patient safety competence on both quality and safety [31]. At least, students reported that in their clinical learning setting, they perceived their impact on patient care outcomes.

Also, they highlighted the need for a more combined approach to theoretical and clinical education such as, supportive learning environments with concentration on systems-based approaches to prevent errors, based on team work and systematically learning from and preventing errors [2,12]. Despite, a remarkable relapse in working in teams and management of adverse events are identified during the progress of the academic nursing program [9].

Cruz et al. [31] argues that as the nursing students advance to a higher level of nursing education, their patient safety competence both in the classroom and clinical settings declines. Similarly, the study of Tella et al. [2] revealed that unfavourable experiences may reflect final year nursing students' decreasing levels of confidence related to patient safety. It can also be drawn that their academic level in the nursing program is one of the factors that influences their developed patient safety competence as students. Just as observing mentors and staff members, who do not always represent exemplary behaviour. The poor role models, and nursing students' sense of belonging in clinical settings have an adverse impact on the students' learning $[2,23]$.

Furthermore, the combination of academic learning and clinical practice supports the mastery of patient safety skills $[3,10]$.

Vaismoradi et al. [12] argues, the nurse education, in both academic and clinical settings, can serve as a role model for socialisation and can enhance student willingness to collaborate and teach one another. Thus, in order to allow nursing students to learn how to prevent and handle adverse events it is important that they are able to work with experts from both academic and clinical setting [16]. However, this is still an area needing further study to better understand the relationship between these two variables.

The present study has some limitations. Study sample were final years nursing students within one University of Applied Sciences in the Netherlands, which had only a 40 -week internship. Moreover, only $18 \%$ did internship in an university hospital. Nevertheless, this study provided to the current body of knowledge about patient safety competence of nursing students. More particularly, these findings contributed to the limited literature about patient safety competence of nursing students in the Netherlands.

\section{Conclusion}

It may be concluded that the course on Innovation and Policy of Safety management has a significant effect on the mastery of patient 
safety competencies. The course positively affected perceived level of mastery of a 'patient safety culture', 'more effective communication' and 'optimizing human and environmental factors'. Overall, the course had a significant effect on the total score in management of the patient safety competencies.

Further research should be conducted where patient safety is embedded as a specific course in the entire Bachelor nursing curriculum. Both, to achieve continuous improvement in patient safety and quality of care, as minimization errors and the advance of fundamental skills.

\section{Conflict of interest}

None declared.

\section{References}

1. Mansour M (2015) Factor analysis of nursing students' perception of patient safety education. Nurse Education Today 35: 32-37.

2. Tella S, Smith N, Partanen P, Turunen H (2015) Learning Patient Safety in Academic Settings: A Comparative Study of Finnish and British Nursing Students' Perceptions. J Clin Nursing 24: 2954-2964.

3. Berndt J (2014) Patient safety and simulation in prelicensure nursing education: An integrative review. Teaching and Learning in Nursing 9: 16-22.

4. Thornlow D, McGuinn K (2010) A necessary sea change for nurse faculty development: spotlight on quality and safety. J professional nursing 26: 1-81.

5. Hughes R (2008) Patient safety and quality: An evidence-based handbook for nurses. Agency for Healthcare Research and Quality Publication 8: 0043.

6. Wakefield A, Attree M, Braidman I, Carlisle C, Johnson M et al. (2005) Patient safety: do nursing and medical curricula address this theme? Nurse Education Today 25: 333340

7. Vaismoradi M, Salsali M, Marck P (2011) Patient safety: nursing students' perspectives and the role of nursing education to provide safe care. International Nursing Review 58: 434-442.

8. Kiersma ME, Plake KS, Darbishire PL (2011) Patient safety instruction in US health professions education. Am J Pharm Educ 75: 162. [Crossref]

9. Lukewich J, Edge D, Tranmer J, Raymond J, Miron J, et al. (2015) Undergraduate baccalaureate nursing students' self-reported confidence in learning about patient safety in the classroom and clinical settings: An annual cross-sectional study (20102013). Int J Nurs Studies 52: 930-938.

10. Tella S, Liukka M, Jamookeeah D, Smith N, Partanen P, et al. (2014) What do nursing students learn about patient safety? An integrative literature review. J Nurs Educ 53: $7-13$.

11. Brand S, Kristen M, Chang Y, Cheng M, Lipinski C, et al. (2015) Team strategies and tools to enhance performance and patient safety training: The effect of training on both nursing staff perceptions regarding physician behaviors and patient satisfaction scores in the ED. J Hosp Admin 4: 49-52.

12. Vaismoradi M, Griffiths P, Turunen H, Jordan S (2016) Transformational leadership in nursing and medication safety education: a discussion paper. Journal of Nursing Management.
13. Wagner C, de Bruijne MC, Zegers M, Hoonhout LHF, Christiaans I, et al. (2005) Rapportage Pilot Studie. VU Medisch Centrum / EMGO Instituut/ NIVEL.

14. Jeffrey M, Rothschild M, Christopher P, Landrigan M, John W, et al. (2005) The Critical Care Safety Study: The incidence and nature of adverse events and serious medical errors in intensive care. Critical Care Medicine 33: 1694-1700.

15. Miltner R, Patrician P, Dawson M, Jukkala A (2012) Incorporating quality and safety education into a nursing administration curriculum. The J Nurs Administ 42: 478-482.

16. Lee N, Jang H, Park S (2016) Patient safety education and baccalaureate nursing students' patient safety competency: a cross-sectional study Nursing \& Health Sciences. 18: 163-171.

17. Ranjbar H, Emami Zeydi A (2016) Patient Safety: An important yet Neglected issue in Nursing Education J Patient Safety.

18. Ginsburg L, Tregunno D, Norton P, Smee S, de Vries I, et al. (2015) Developmen and testing of an objective structured clinical exam (OSCE) to assess socio cultural dimensions of patient safety competency. Britisch Med J Quality \& Safety 24: 188-194.

19. Ginsburg L, Castel E, Tregunno D, Norton P (2015) The H-PEPSS: an instrument to measure health professionals' perceptions of patient safety competence at entry into practice. Britisch Med J Quality \& Safety 21: 676-684.

20. Okuyama A, Martowirono K, Bijnen B (2011) Assessing the patient safety competencies of healthcare professionals: a systematic review. Britisch Med J Quality \& Safety 20: 991-1000.

21. Madhavanprabhakaran G, Al-Khasawneh E, Ramasubramaniam S, Akintola A (2015) Students' perceived benefits of pre-clinical simulation based training (PSBT) on enhancing patient safety and clinical learning outcomes. Nurse Education in Practice 15: 3.

22. Bianchi M, Bressan V, Cadorin L, Pagnucci N, Tolotti A, et al. (2016) Patient safety competencies in undergraduate nursing students: a rapid evidence assessment. $J A d v$ Nurs 72: 2966-2979. [Crossref]

23. Abbott A, Fuji K, Galt K, Paschal K (2002) How baccalaureate nursing students value an interprofessional patient safety course for professional development. Intern Scholary Research Notices Nursing 12: 401358.

24. Tregunno D, Ginsburg L, Clarke B, Norton P (2014) Integrating patient safety into health professionals' curricula: a qualitative study of medical, nursing and pharmacy faculty perspectives. Britisch Med J Quality \& Safety 23: 257-264.

25. Sullivan D, Hirst D, Cronenwett L (2009) Assessing quality and safety competencies of graduating prelicensure nursing students. Nursing outlook 57: 323-331.

26. Smith EL, Cronenwett L, Sherwood G (2007) Current assessments of quality and safety education in nursing. Nurs Outlook 55: 132-137. [Crossref]

27. Mansour M (2013) Examining patient safety education in pre-registration nursing curriculum: Qualitative study. J Nurs Educ Pract 3: 157- 167.

28. Vaismoradi M, Bondas T, Jasper M, Turunen H (2014) Nursing students' perspectives and suggestions on patient safety-implications for developing the nursing education curriculum in Iran. Nurse Education Today 34: 265-270.

29. Stevenson E, Chudgar S, Turner K, Molloy M, Phillips B, et al. (2015) How We Engage Graduating Professional Students in Interprofessional Patient Safety. Nursing Forum.

30. Tingle J (2011) The WHO Patient Safety Curriculum Guide. British J of Nurs 20: 1456-1457.

31. Cruz J, Colet C, Cruz C, Al-otaibi J, Qubeilat H, et al. (2015) Patient Safety Competence of Nursing Students in Saudi Arabia: A Self-Reported Survey International Journal of Health Sciences 9: 4.

Copyright: (C2017 Olaf T. This is an open-access article distributed under the terms of the Creative Commons Attribution License, which permits unrestricted use, distribution, and reproduction in any medium, provided the original author and source are credited. 